\title{
Costs and gains of complex procedures to rehabilitate end stage ocular surface disease
}

\author{
G Geerling, C S C Liu, J R O Collin, J K G Dart
}

Br J Ophthalmol 2002;86:1220-1221

Background: The management of patients with end stage ocular surface disease sometimes requires extensive surgical treatment that can only be provided by specialised ophthalmology. The authors discuss the costs and gains of such complex techniques based on a case of bilateral corneal blindness secondary to Stevens-Johnson syndrome.

Methods and results: Despite multiple lid surgery for trichiasis and repeated corneal grafting for perforations a white female patient became bilaterally blind at the age of 29 years. She also suffered from disabling discomfort in the right dry eye. At the age of 52 a two stage osteo-odonto-keratoprosthesis achieved visual rehabilitation in the left eye and microvascular transplantation of an autologous, submandibular gland resulted in sufficient lubrication to alleviate her severe discomfort in the right eye. As a result of these procedures she was able to take up a regular job again. The total costs of rehabilitation were $£ 13661$ which compare with annual gains and regains for society of $£ 13497$. An additional $£ 4625$ was saved annually in guide dog costs.

Conclusion: This estimate shows that despite the expense of these complex techniques gains are made well within the second year after rehabilitation. In view of the benefit in quality of life for the patient and monetary savings for society these procedures should be funded by national health services at specialist centres.

O cular surface disease and absolute tear deficiency can lead to corneal blindness and severe discomfort. Conventional corneal surgery in this situation often fails. More complex approaches, usually requiring extensive specialist surgical skills and theatre resources are available but health authorities may be reluctant to provide appropriate funding for these. The cost implications of the two most extensive surgical procedures in ocular surface rehabilitation are estimated for a case with severe ocular surface disease associated with bilateral corneal blindness and severe discomfort. These costs are balanced against the potential savings for society. The economic analysis of this case has been presented to assist in the decision making process for hospital service providers and health authorities faced with these blind patients who may require apparently expensive procedures.

\section{METHODS AND RESULTS}

A female white child suffered from Stevens-Johnson syndrome following prophylactic phenobarbitone ingestion at the age of 5. She developed entropion, progressive ocular surface disease, and absolute tear deficiency. This resulted in severe discomfort as well as recurrent episodes of microbial keratitis leading to a bilateral, progressive corneal pannus and blind registration at the age of 29 . In the subsequent 33 years she was treated with intensive medical therapy for dry eye, silicone rubber contact lenses and underwent 15 ophthalmic procedures including multiple adnexal surgery for entropion, aspiration of a cataractous lens, cyclodestruction to treat secondary angle closure glaucoma, four corneal and one limbal stem cell graft to treat corneal perforation and surface failure. All procedures failed to achieve visual rehabilitation or relief from the severe right dry eye.

In 1998-9 rehabilitation was achieved by means of two complex procedures which both require surgeons with specialist training in these procedures. Relief from severe discomfort and freedom from applying lubricants every 5-10 minutes was achieved by means of an autologous submandibular gland (SMG) transplant to lubricate the dry right eye. ${ }^{1}$ This involved an ophthalmic surgeon and a microvascular maxillofacial surgeon. Theatre time for this one stage procedure was 7 hours, which is normal for this type of surgery. Visual rehabilitation was finally achieved by means of a two stage osteo-odontokeratoprosthesis (OOKP) in 1999. The two OOKP stages involved one maxillofacial surgeon for 4 hours and one ophthalmic surgeon for a total of approximately 10 hours operating time plus a set of special instruments. This gave the patient a vision of $6 / 6$ and a visual field span of $100^{\circ}$. The patient subsequently became independent from her guide dog and pharmaceutical tear substitutes. She has worked for the past 2 years in a regular job and was removed from the blind register.

Costs and annual gains due to surgical rehabilitation of this patient were estimated for the year 1998-9. Moorfields Eye Hospital/UCL/London is currently the only provider of SMG transplantation in the United Kingdom as is Sussex Eye Hospital/Brighton for OOKP. The figures provided by these hospitals were therefore used for the costs/gains calculations. The costs for SMG transplantation were based on an average operating time of 7 hours at $£ 836.7 /$ hour at Moorfields Eye Hospital including all preoperative and postoperative inpatient assessment and care. The costs for the two stages of OOKP surgery were previously calculated as an itemised estimate by Brighton Healthcare/NHS. The costs for all follow up examinations during the first postoperative year were also given by the two providing hospitals. The total costs of surgical rehabilitation were $£ 13661$ (Table 1).

Major financial gains for society were identified by the end of benefit for blindness at $£ 103 /$ week and tax paid by the patient when restarting a job. For the latter an average national salary of $£ 20800$ was assumed. Figures were provided by the Royal National Institute for the Blind (RNIB). Additional savings resulted from ending the need for pharmaceutical tear substitutes and a guide dog. As patients who undergo submandibular gland transplantation often need one bottle of unpreserved tear substitute per day, costs of pharmaceutical tear substitutes were calculated at the pharmacy price of Moorfields Eye Hospital for one bottle of unpreserved hypromellose per day. The total life time costs as provided by Guide Dog for the Blind/UK were $£ 37000$ per dog, with a maximum working life of 8 years. The total annual gain for society by the ending of benefit and need for lubricants as well as tax paid was $£ 13497$ (Table 2 ). The total potential gain 
Table 1 Costs of rehabilitation and aftercare for 1 year

\begin{tabular}{lr}
\hline Type of costs & Amount $(£)$ \\
\hline Costs of submandibular gland transplantation * & 5857 \\
Procedure of 7 hours $\times £ 836.7 /$ hour & 270 \\
6 peroperative out patient visits for 1 year $\times £ 45 /$ visit & 3388 \\
Costs of osteo-odonto-keratoprosthesis & 3326 \\
Procedure stage 1 & 550 \\
Procedure stage 2 & 270 \\
Materials (optical cylinder and others) & 13661 \\
10 peroperative out patient visits for 1 year $\times £ 27 /$ visit & \\
Total costs (£) &
\end{tabular}

Table 2 Annual potential savings due to rehabilitation

\begin{tabular}{llr}
\hline Type of gains/regains & & Amount (£) \\
\hline Benefit for blindness & $£ 103 /$ week $\times 52^{*}$ & 5356 \\
Additional tax revenue & $33 \%$ tax on $£ 20800^{*}$ & 6864 \\
Pharmaceutical tear substitutes & $£ 3.5 / 10 \mathrm{ml}$ unpreserved hypromellose $\dagger \times 365$ & 1277 \\
Guide dog & $£ 37000 /$ life time of a guide dog $\ddagger$ & 4625 \\
Total costs $(£)$ & & 18122 \\
\hline
\end{tabular}

*Figures according to Royal National Institute for the Blind.

†Figures according to pharmacy department of Moorfields Eye Hospital.

‡Figures according to Guide Dogs for the Blind.

including the annual costs for a guide dog of $£ 4625$ was $£ 18122$ per year.

\section{DISCUSSION}

Although absolute figures will change, the ratio between costs and potential savings of rehabilitation will be relatively stable in the long term. Our estimates compare well with other published data on the costs of blindness, such as benefits. ${ }^{2}$ We are aware that our figures give an estimate only, but according to our cost/ benefit calculations gains are made in the second year after rehabilitation. Given this very clear margin of gain we did not take further the minor additional costs and gains, such as costs of specialist surgical training and gains from avoiding hospital travel and additional contribution towards the national insurance scheme. Also, our calculations do not take into consideration potential costs for the management of complications (for example, for epiphora other salivary gland transplantion) or recurrent loss of rehabilitation (for example, in the case of extrusion of the OOKP), but similar costs might be expected in the natural course of the disease.

As the peak incidence of Stevens-Johnson syndrome is in the second and third decade of life it often results in blindness at a young age, with the majority of working life still to come. Early rehabilitation therefore offers a substantial potential for financial gains for society as well as the individual patient. Based on the cost/gain ratio shown here rehabilitation with a subsequent 30 years of working of life would require a minimum success rate of $3.4 \%$ for both procedures combined to result in financial gains. In trained hands SMG transplantation and OOKP have both been reported to have a success rate of over $75 \% .{ }^{3}{ }^{4}$ From this it can be estimated that a financial gain would result well within the second year of rehabilitation. Although complex procedures for the rehabilitation of blindness and severe discomfort due to ocular surface disease initially appear to be cost intensive they offer substantial saving potential.

A parameter difficult to put into monetary terms is the impact of rehabilitation on quality of life. Given the amount of work available on the impact of rehabilitation from pain or blindness on quality of life it is not necessary to labour this point. ${ }^{5-8}$ In this light and in view of the monetary savings shown here, it can be concluded that complex procedures should be supplied by national health services at specialist centres. We hope that this report will inform hospital and health authority decision making.

\section{ACKNOWLEDGEMENTS}

We thank Mrs Hazel Hopkins and Mr Ian Coldrick (BrightonHealthcare/NHS Trust), Mr Richard Wormald (Department of Epidemiology/Moorfields Eye Hospital), and Mr Ian Knott and $\mathrm{Mr}$ Patrick O'Sullivan (Department of Finance/Moorfields Eye Hospital/ London) for their advise regarding the cost calculations.

This work received its funding from the German Research Council (DFG: Ge 895/4-1), the Special Trustees of Moorfields Eye Hospital, and the NHS executive; the views expressed in this publication are those of the authors and not necessarily those of the funding bodies executives.

\section{Authors' affiliations}

G Geerling, Medizinische Universität Lübeck, Ratzeburger Allee 160, 23538 Lübeck, Germany

J R O Collin, J K G Dart, Moorfields Eye Hospital, 162 City Road, London ECIV 2PD, UK

C S C Liu, Sussex Eye Hospital, Eastern Road, Brighton BN2 5BF, UK

Correspondence to: Dr Gerd Geerling, Department of Ophthalmology, University Hospital, Lübeck, Ratzeburger Allee 160, 23538 Lübeck, Germany; ggeerling@ophtha.mu-luebeck.de

Accepted for publication 15 May 2002

\section{REFERENCES}

1 Geerling G, Sieg P, Bastian GO, et al. Transplantation of the autologous submandibular gland for most severe cases of keratoconjunctivitis sicca. Ophthalmology 1998; 105:327-35

2 Matz H, Falk M, Gottinger W, et al. Cost-benefit analysis of diabetic eye disease. Ophthalmologica 1996;210:348-53.

3 Liu C, Sciscio A, Smith G, et al. Indications and technique of modern osteo-odonto-keratoprosthesis (OOKP) surgery. eyenews 1998;4:17-22.

4 Geerling G, Sieg P, Meyer C, et al. [Transplantation of autologous submandibular glands in very severe keratoconjunctivitis sicca. 2 year outcome.] Ophthalmologe 1998;95:257-65.

5 Hirsch JD, Kozma CM, Wojcik AR, et al. Economic and quality of life impact of dry eye symptoms: A sjoegren's sydnrome patient sruvey. Invest Ophthalmol Vis Sci 1998;39(Suppl):S

6 Elliot JL, Kuyk TK. Self-reported functional and psychosocial outcomes of blind rehabilitation. J Visual Imparim Blindness 1994;88:206-12.

7 Desai $\mathbf{P}$, Reidy A, Minassian DC, et al. Gains from cataract surgery: visual function and quality fo life. Br J Ophthalmol 1996;80:868-73.

8 Szlyk JP, Arditi A, Bucci P, et al. Self-report in functional assessment of low vision. J Visual Impairm Blindness 1990;84:61-6. 